\title{
Impact of Community Education on Antenatal Care Visits in Bangladesh: A Multilevel Analysis
}

\author{
Md. Morshadur Rahman* and Md. Akhtar Hossain \\ Department of Statistics, Dhaka University, Dhaka-1000, Bangladesh
}

(Received: 2 October 2018 ; Accepted: 5 December 2018)

\begin{abstract}
Antenatal care (ANC) utilization has a great role in reducing pregnancy-related complications and maternal mortality. Besides individual demographic and socio-economic factors, community characteristics can influence a mother's decision to seek antenatal care visits. This study makes an attempt to determine the influence of community education on adequate ANC visits in Bangladesh by using Bangladesh Demographic and Health Survey (BDHS) 2014 data. Multilevel logistic regression model has been employed for the purpose of the analysis. Study findings showed a significant positive association of community education on adequate ANC visits. Other community and individual level covariates such as place of residence, region of residence, birth order, mother's age at birth, household wealth, media access, husband's education, decision making on health care also showed significant association with adequate ANC visits.
\end{abstract}

Keywords: Antenatal care, ANC visits, Community education, Multilevel logistic regression, Bangladesh.

\section{Introduction}

Since women constitute nearly half of the population of the world, maternal health has a great importance on human development and it is a crucial part of the general health. Pregnancy and childbirth are often related to complications and sometimes these complications become life-threatening. Between 1990 and 2015, maternal mortality has fallen by $44 \%$ worldwide ${ }^{1}$. But unfortunately, the situation has not improved in developing countries. Due to pregnancy and delivery related complications, approximately 830 maternal deaths occur every day in the current world and $99 \%$ of these deaths occur in developing countries ${ }^{1}$. More than $50 \%$ of maternal deaths occur in sub-Saharan Africa and 33\% occur in south Asian countries ${ }^{2}$. The maternal mortality ratio (MMR) significantly decreased between 2001 and 2010, from 322 to 194 deaths per one thousand live births in Bangladesh $^{3-4}$. But the findings from Bangladesh Maternal Mortality Survey (BMMS) 2016 indicates that the MMR has stalled in recent years implying that the targets of 121 per 100,000 live births by 2022 aimed by fourth Health, Population and Nutrition Sector Program (HPNSP) and the Sustainable Development Goal (SGD) 3 goal of decreasing MMR to 70 deaths per 100,000 live births by 2030 will be facing a huge challenge to be achieved ${ }^{5-6}$. Antenatal care (ANC) helps women to prepare themselves for delivery and to understand pregnancy and childbirth related complications and ANC interventions can reduce maternal deaths significantly ${ }^{7-8}$.

The number of ANC visits during pregnancy has great importance in reducing complications and maternal mortality ${ }^{9}$. The minimum number of ANC visits proposed by the World Health Organization (WHO) is 4 and the HPNSDP framework had set a goal of achieving nearly half of the pregnant women seeking minimum 4 ANC visits by $2016^{10}$. In Bangladesh, this adequate number of ANC visits significantly increased from $17 \%$ to $31 \%$ between 2004 and $2014^{11}$. But still, the rate is far behind from the target. Several factors are associated with the number of ANC visits women seek during pregnancy. Mother's age at birth has significant impact on women's ANC visits behavior ${ }^{12}$. Women from rich families had higher rate of ANC visits than the women from poor families ${ }^{13}$. Order of the birth have been found as another important factor as women are more likely to seek complete antenatal care visits for first birth than for higher order births ${ }^{14}$. Moreover, women reside in urban area were more likely to have at least 4 ANC visits than mother from rural areas ${ }^{15}$. Mother's education has been reported as the most consistent and essential factor of the maternal health care seeking behavior ${ }^{16}$. Educated mothers were found more in number in receiving 4 or more ANC visits than uneducated mothers ${ }^{15}$. Many studies on maternal health support that husband's occupation, women's employment, autonomy, parity, mass media access, NGO or community clinic access etc. have significant impact on ANC utilization ${ }^{17-20}$.

Health care seeking is not only dependent on mother's individual characteristics, but also on some community characteristics such as social features and location of health care services ${ }^{21}$. However, a review of related literature shows that the number of studies that focus on examining the determinants of maternal health at community level are very scarce in Bangladesh. As mother's individual level education has huge positive impact on ANC visits, this study makes an attempt to examine whether community education has an influence on ANC visits in Bangladesh.

\section{Data and Methods}

Data

Bangladesh Demographic and Health Survey (BDHS) $2014^{9}$, a nationwide survey data has been utilized to conduct this study. A total of 18000 residential households were selected for the interview and BDHS successfully interviewed 17863 ever married women aged 12-49 years. For the purpose of the analysis, ANC information of women has been assessed and if a woman had two or more live births, this analysis refers to the most recent live birth

*Author for correspondence. e-mail: morshed87@ du.ac.bd 
only. Finally, a total of 3,975 women were selected who gave birth within 3 years preceding the survey for the purpose of the analyses.

\section{Variables}

According to WHO, the care given to women during their pregnancy by skilled health-care professionals is termed as antenatal care $^{2}$. In this study, the response is a binary variable coded as 1 for the complete ANC visits (ANC visits greater or equal 4) and 0 for incomplete $A N C$ visits (ANC visits less or equal 3). Socio-economic, demographic, and community level factors have been considered as explanatory variables according to reviews of related literature ${ }^{12-20,22-25}$. The explanatory variables included are age (in years) of mothers at last birth $(<20,20-29,>29)$, birth order of the last child (1, 2-3, 4 and above), wealth index (poor, middle, rich), religion (muslim, non-muslim), education of mother (none, primary, secondary, higher), husband's education (none, primary, secondary, higher), media access (yes, no), awareness of community clinic (yes, no), and mother's involvement in decision making on health care (involved, not involved).

Three community characteristics; place of residence (urban and rural), region of residence (Sylhet, Barisal, Chittagong, Dhaka, Khulna, Rajshahi and Rangpur) and community education are considered in this study. Community education is constructed as the proportion of mother who completed secondary and higher level of education in the primary sampling unit. This proportion is then divided into terciles and classified as low, medium and high.

\section{Statistical Methods}

The Chi-Square test of association has been used to determine the unadjusted association between antenatal care and selected individual and community characteristics. Multilevel logistic regression model has been employed to find out the adjusted effects of both individual and community level variables on ANC visits.

BDHS 2014 data are based on multistage stratified cluster sampling procedure. So, it follows a hierarchical data structure which introduces multilevel dependency or correlation among the observations. Thus, a two-level multilevel logistic regression model has been applied in this study where the units at lower level (level 1) are individuals (ever married women aged 12-49) who are nested within units at higher level (level 2) which are clusters or communities.

The specified two-level multilevel logistic regression model is

$\operatorname{logit}\left(\pi_{i j} \mid u_{0 j}, e_{i j}\right)=\log \left[\frac{\pi_{i j}}{1-\pi_{i j}}\right]=\beta_{0}+\beta_{1} X_{1 i j}+\beta_{2} X_{2 i j}+$ $\cdots+\beta_{n} X_{n i j}+u_{0 j}+e_{i j}$

where $\pi_{i j}$ is the probability of complete ANC visits of a woman $i$, residing in a community $j ; \beta_{0}$ is the log odds of the intercept; $\beta_{1}, \ldots \ldots \beta_{n}$ are the coefficients of individual and community level variables; $X_{1 i j} \ldots . X_{n i j}$ are individual and community level variables; $u_{0 j}$ is random error at community levels; $e_{i j}$ is random error at individual levels.

Four models have been estimated in this analysis. Model 1 was a null model with no explanatory variable. Model 2 used only is the main exposure variable: community education. Model 3 included all the community level explanatory variables. Model 4 included both individual and community level explanatory characteristics. The individual level explanatory variable "Mother's education" was dropped in the final model (Model 4) to avoid possible collinearity issues since the community level variable 'Community education' was derived from mother's individual educational attainments.

The estimated fixed effects were expressed as odds ratios (OR) and the random effects were expressed as intra-class correlation (ICC) ${ }^{25}$. STATA 12.1 has been used to carry out the analyses.

\section{Results}

\section{Univariate Analysis}

It was observed from the sample that most of the women $(57 \%)$ belonged to the age group $20-29$, while $27.2 \%$ of women gave birth at ages less than 20 years and $15.8 \%$ of women's ages at last birth were 30 years or more. Nearly half of the women $(46.3 \%)$ had given birth to 2 to 3 children, whereas $39.3 \%$ women had only 1 child. $19.2 \%$ women in the sample belonged to middle class family, while percentages of women from poor and rich family were almost even (40.1\% and $40.7 \%$ respectively). Most of the women in the sample were Muslim (91.9\%) in religion. Almost half of the mothers in study sample (47.2\%) had completed their secondary education, while $13.8 \%$ mothers had no education and $27.4 \%$ mothers completed primary education. The number of women who completed their higher education was very low (11.6\%). It was seen that the percentages of primary and secondary education completed husbands were $30.3 \%$ and $30.9 \%$ respectively.

Further more, percentage of uneducated and higher educated husbands were $23.2 \%$ and $15.6 \%$ respectively. A major proportion $(62.4 \%)$ of women had media access and most of the women $(91.1 \%)$ in the study sample were not aware of the existence of community clinics. More than one-third $(39.8 \%)$ of the women had no involvement in decision making on their own health care. Women's community characteristics revealed that $67.3 \%$ of the women in the sample came from rural areas. In addition, highest proportion of the women (19\%) came from Chittagong region and lowest proportion (11.5\%) came from Khulna region. Only $20 \%$ of women reside in communities with low proportion of educated women while this percentage were $40.2 \%$ and $39.1 \%$ who reside in communities with medium and high proportion of educated women. Out of the 3,975 women in study sample, only $32 \%$ women had completed ANC visits during their pregnancy. 


\section{Bivariate Analysis}

Table 1 presents the Chi-square tests of associations with cross percentage distributions to determine whether the explanatory variables exhibited associations with ANC visit behavior of the mothers in study sample.

Table 1. Percentage distribution of women by number of ANC visits and selected explanatory variables.

\begin{tabular}{|c|c|c|c|c|}
\hline \multirow[t]{2}{*}{ Characteristics } & \multicolumn{2}{|c|}{$\begin{array}{c}\text { Antenatal care } \\
\text { visits }\end{array}$} & \multirow[t]{2}{*}{$\begin{array}{l}\text { No. of } \\
\text { women }\end{array}$} & \multirow[t]{2}{*}{$p$-value } \\
\hline & $\begin{array}{l}\text { Visits } \\
\leq 3\end{array}$ & $\begin{array}{l}\text { Visits } \\
\geq 4\end{array}$ & & \\
\hline \multicolumn{5}{|l|}{$\begin{array}{l}\text { Mother's age at } \\
\text { last birth }\end{array}$} \\
\hline$<20$ years & 68.9 & 31.1 & 1081 & \\
\hline 20-29 years & 66.3 & 33.7 & 2267 & 0.012 \\
\hline$>29$ years & 72.4 & 27.6 & 627 & \\
\hline \multicolumn{5}{|l|}{ Birth order } \\
\hline 1 & 62.0 & 38.0 & 1564 & \\
\hline $2-3$ & 68.5 & 31.5 & 1840 & $<0.001$ \\
\hline 4 and above & 82.8 & 17.2 & 571 & \\
\hline \multicolumn{5}{|l|}{ Wealth } \\
\hline Poor & 81.3 & 18.7 & 1595 & \\
\hline Middle & 75.2 & 24.8 & 762 & $<0.001$ \\
\hline Rich & 51.5 & 48.5 & 1618 & \\
\hline \multicolumn{5}{|l|}{ Religion } \\
\hline Muslim & 68.5 & 31.5 & 3655 & 0.039 \\
\hline Non-Muslim & 62.8 & 37.2 & 320 & \\
\hline \multicolumn{5}{|l|}{ Education } \\
\hline No & 85.4 & 14.6 & 549 & \\
\hline Primary & 78.3 & 21.7 & 1091 & $<0.001$ \\
\hline Secondary & 64.7 & 65.3 & 1875 & \\
\hline Higher & 36.1 & 63.9 & 460 & \\
\hline \multicolumn{5}{|l|}{$\begin{array}{l}\text { Husband's } \\
\text { education }\end{array}$} \\
\hline No & 81.2 & 18.8 & 922 & \\
\hline Primary & 76.2 & 23.8 & 1204 & $<0.001$ \\
\hline Secondary & 62.3 & 37.7 & 1230 & \\
\hline Higher & 43.6 & 56.4 & 619 & \\
\hline \multicolumn{5}{|l|}{ Media access } \\
\hline Yes & 59.2 & 40.8 & 2480 & $<0.001$ \\
\hline No & 82.6 & 17.4 & 1495 & \\
\hline \multicolumn{5}{|l|}{$\begin{array}{l}\text { Aware of } \\
\text { community } \\
\text { clinic }\end{array}$} \\
\hline Yes & 74.1 & 25.9 & 352 & 0.01 \\
\hline No & 67.4 & 32.6 & 3623 & \\
\hline \multicolumn{5}{|l|}{$\begin{array}{l}\text { Decision on } \\
\text { Health care }\end{array}$} \\
\hline Respondent & 65.2 & 34.8 & 2393 & $<0.001$ \\
\hline
\end{tabular}

\begin{tabular}{lcccc}
$\begin{array}{l}\text { involved } \\
\text { Respondent not }\end{array}$ & 72.2 & 27.8 & 1582 & \\
$\begin{array}{l}\text { Involved } \\
\text { Place of } \\
\text { residence }\end{array}$ & & & & \\
Urban & 54.0 & 46.0 & 1298 & $<0.001$ \\
Rural & 74.8 & 25.2 & 2677 & \\
Region of & & & & \\
residence & & & & \\
Sylhet & 77.7 & 22.3 & 609 \\
Barisal & 74.2 & 25.8 & 480 \\
Chittagong & 71.4 & 28.6 & 755 \\
Dhaka & 64.6 & 35.4 & 684 \\
Khulna & 59.6 & 40.4 & 456 \\
Rajshahi & 69.2 & 30.8 & 490 \\
Rangpur & 56.3 & 43.7 & 501 \\
Community & & & & \\
Education & & & \\
Low & 85.4 & 14.6 & 821 \\
Medium & 69.3 & 30.7 & 1598 \\
High & 57.5 & 42.5 & 1556 \\
\hline
\end{tabular}

It is clear from Table 1 that women aged 20-29 years were more in numbers to seek complete ANC visits than other two age groups. Child's birth order was found negatively associated with complete ANC visits while there was an observed positive association between completing ANC visits and household wealth. It was also seen that proportion of complete ANC visits among non-Muslim mothers $(37.2 \%)$ were higher than Muslims mothers (31.5\%). Mother's education has exhibited a significant positive relationship with complete ANC visits. Similar differential has also been observed for husband's education. Moreover, access to media and women's decision making about their health had significant positive impact on adequate ANC visits, whereas community clinic awareness was inversely related to complete ANC visits. Statistically significant association has also been observed for place and region of residence with complete ANC utilization. Furthermore, women who lived in a highly educated community were more in numbers to seek adequate ANC than the women who lived in a community with medium and lower proportion of educated women. It was clear from Table 1 that all covariates were significantly associated with complete ANC utilization.

\section{Multilevel Analysis}

The results of multilevel logistic regression models are summarized in Table 2. The Model 1 with no covariates showed significant variation on complete ANC visits across the communities $(\tau=1.23, p<0.01)$. Intra-class correlation coefficient was found to be $27.1 \%$ presenting the variability on ANC visits due to community level. 
Table 2. Multilevel logistic regression model estimated odds ratios (95\% confidence intervals) of selected community and individual level factors on ANC visits.

\begin{tabular}{|c|c|c|c|c|}
\hline Variables & $\begin{array}{l}\text { Model } 1 \\
\text { Empty model }\end{array}$ & $\begin{array}{l}\text { Model } 2 \\
\text { Community education } \\
\text { model }\end{array}$ & $\begin{array}{l}\text { Model } 3 \\
\text { All community } \\
\text { variables model }\end{array}$ & $\begin{array}{l}\text { Model } 4 \\
\text { Individual/ Community } \\
\text { variables model }\end{array}$ \\
\hline & OR $(95 \% \mathrm{CI})$ & OR $(95 \% \mathrm{CI})$ & OR $(95 \% \mathrm{CI})$ & OR $(95 \% \mathrm{CI})$ \\
\hline \multicolumn{5}{|l|}{ Fixed Effects } \\
\hline \multicolumn{5}{|c|}{ Individual Characteristics } \\
\hline \multicolumn{5}{|l|}{ Mother's age at last birth } \\
\hline$<20$ years & & & & 1.00 \\
\hline 20-29 years & & & & $1.31(1.04,1.65)^{* *}$ \\
\hline$>29$ years & & & & $1.24(0.88,1.75)$ \\
\hline \multicolumn{5}{|l|}{ Birth order } \\
\hline 1 & & & & 1.00 \\
\hline $2-3$ & & & & $0.69(0.56,0.86)^{* * *}$ \\
\hline 4 and above & & & & $0.52(0.37,0.75)^{* * *}$ \\
\hline \multicolumn{5}{|l|}{ Wealth } \\
\hline Poor & & & & $0.97(0.75,1.24)$ \\
\hline Middle & & & & 1.00 \\
\hline Rich & & & & $2.00(1.58,2.54)^{* * *}$ \\
\hline \multicolumn{5}{|l|}{ Religion } \\
\hline Muslim & & & & $0.87(0.64,1.18)$ \\
\hline Non-Muslim & & & & 1.00 \\
\hline \multicolumn{5}{|l|}{ Husband's education } \\
\hline No & & & & $0.75(0.58,0.97)^{* *}$ \\
\hline Primary & & & & $0.74(0.6,0.92)^{* * *}$ \\
\hline Secondary & & & & 1.00 \\
\hline Higher & & & & $1.53(1.21,1.94)^{* * *}$ \\
\hline \multicolumn{5}{|l|}{ Media access } \\
\hline Yes & & & & $1.58(1.27,1.95)^{* * *}$ \\
\hline No & & & & 1.00 \\
\hline \multicolumn{5}{|l|}{ Aware of community clinic } \\
\hline Yes & & & & $0.84(0.62,1.11)$ \\
\hline No & & & & 1.00 \\
\hline \multicolumn{5}{|l|}{ Decision on Health care } \\
\hline Respondent involved & & & & $1.30(1.1,1.54)^{* * *}$ \\
\hline Respondent not Involved & & & & 1.00 \\
\hline \multicolumn{5}{|c|}{ Community Characteristics } \\
\hline \multicolumn{5}{|l|}{ Place of residence } \\
\hline Urban & & & $2.52(2.04,3.12)^{* * *}$ & $1.53(1.22,1.91)^{* * *}$ \\
\hline Rural & & & 1.00 & 1.00 \\
\hline \multicolumn{5}{|l|}{ Region of residence } \\
\hline Sylhet & & & $0.68(0.45,1.02)^{*}$ & $0.65(0.43,0.99)^{* *}$ \\
\hline Barisal & & & $0.62(0.42,0.93)^{* *}$ & $0.65(0.45,1.01)^{*}$ \\
\hline Chittagong & & & $0.64(0.45,0.92)^{* *}$ & $0.58(0.39,0.8)^{* * *}$ \\
\hline Dhaka & & & $0.91(0.63,1.29)$ & $0.75(0.51,1.05)^{*}$ \\
\hline Khulna & & & 1.00 & 1.00 \\
\hline Rajshahi & & & $0.71(0.48,1.04)^{*}$ & $0.69(0.47,1.03)^{*}$ \\
\hline Rangpur & & & $1.55(1.07,2.26)^{* *}$ & $1.66(1.14,2.44)^{* * *}$ \\
\hline \multicolumn{5}{|l|}{ Community Education } \\
\hline Low & & 1.000 & 1.00 & 1.00 \\
\hline Medium & & $2.89(2.08,4.02)^{* * *}$ & $2.18(1.59,2.98)^{* * *}$ & $1.66(1.21,2.28)^{* * *}$ \\
\hline High & & $5.23(3.77,7.26)^{* * *}$ & $3.69(2.68,5.08)^{* * *}$ & $1.88(1.35,2.63)^{* * *}$ \\
\hline \multicolumn{5}{|l|}{ Random Effects } \\
\hline \multicolumn{5}{|l|}{ Community Level } \\
\hline Variance (SE) & $1.23 * * *(0.15)$ & $0.86^{* *}(0.123)$ & $0.58 * * *(0.099)$ & $0.53 * * *(0.098)$ \\
\hline $\operatorname{ICC}(\%)$ & 27.1 & 20.8 & 14.9 & 13.9 \\
\hline Log-Likelihood & -2360.574 & -2310.9635 & -2260.9534 & -2135.8572 \\
\hline AIC & 4725.149 & 4629.927 & 4543.907 & 4319.714 \\
\hline BIC & 4737.725 & 4655.078 & 4613.072 & 4470.621 \\
\hline
\end{tabular}

Notes: ${ }^{*} p$ value $<0.1,{ }^{* *} p$ value $<0.05,{ }^{* * *} p$ value $<0.01$

Model 2 revealed statistically significant association of community education with complete ANC visits. The odds of complete ANC visits for women who resided in the communities with high proportion of secondary and higher educated women was more than five times higher than the women who resided in the communities with low portion of 
secondary and higher educated women $(\mathrm{OR}=5.23 ; 95 \% \mathrm{CI}$ : 3.77, 7.26; $p<0.01)$. Similar result was also found for the women who lived in the communities with low proportion of secondary and higher educated women $(\mathrm{OR}=2.892 ; 95 \%$ CI: 3.77, 7.26; $p<0.01)$. Results from random effects showed that variation on ANC visits across communities was still significant $(\tau=0.86, p<0.05)$. The inclusion of community education reduced the intra class correlation from $27.1 \%$ to $20.8 \%$, suggesting that the inclusion improved the overall explained variability in complete ANC visits.

Model 3 included all other community level explanatory variables in addition to community education. Women's place of residence exhibited a significant impact on ANC visits. Women who reside in urban areas had 2.52 times higher odds to seek minimum 4 ANC visits than the women resided in rural region. Statistically significant differences in odds of complete ANC visits were observed by region of residence. When compared to the women in Khulna region, significantly lower odds of having complete ANC visits were observed for women from Barisal $(p<0.05)$ and Chittagong $(p<0.05)$. However, odds of complete ANC visits for women in Rangpur region was higher than the women from Khulna. Community education was again found to have a significant impact on ANC visits after adjusting for additional community level factors. From the estimated random effect showed that adjusting for the additional community level characteristics benefited the Model 3 with reducing variation in complete ANC visits across communities from 0.862 (Model 2) to 0.577 ( $p<0.01$ ).

Being the most complete model, Model 4 used all individual level and community level explanatory variables. It was observed that the odds of complete ANC visits for mothers aged 20-29 years were $31 \%$ higher than the mothers aged < 20 years. Increasing birth order of the child was found to affect mothers' ANC visit completion negatively. In contrast to the first babies, for the babies with birth order 2-3, and 4 and above, women had $30 \%$ and $47.6 \%$ reduced odds respectively to have at least $4 \mathrm{ANC}$ visits. Women from rich families were 2 times as likely as to have at least 4 ANC visits than the women from middle income families. It was also seen that husband's higher education had significant positive impact on their wife's health care behavior.

Women's media access and decision making about their own health care also posed a positive association with their ANC visits. Religion and Community clinic awareness were not found to have any statistically significant effect on women's ANC visits. Like in Model 3, community characteristics have been found significantly related with ANC visits after adjusting for the individual level characteristics. Furthermore, community education again showed positive impact on ANC visits likewise in Model 2 and Model 3. The estimated random effect depicted that community level variance was slightly reduced to 0.530 from $0.577(p<0.01)$ after adjusting for the individual level characteristics in Model 4. Intra class correlation was reduced to $13.9 \%$ from $14.9 \%$ implying that inclusion of individual level variables slightly improved the overall explained variability of ANC visits. The AIC and BIC values from all 4 models suggested that the Model 4 was the best choice to explain women's ANC visit behavior.

\section{Discussion}

Bangladesh is a developing country and like other developing countries, maternal health care utilization rate in Bangladesh is far below from acceptable standard. The principal aim of this study was to disclose how community characteristics, especially how community education affects mothers ANC visits behavior in Bangladesh by using multilevel logistic regression model. In addition, the adjusted effect of community education after controlling for other community and individual level variables was also subject to observe.

Four models with different sets of explanatory factors were estimated. The intra-class correlation from all four models confirmed the significant amount of heterogeneity on ANC visits across communities. Model 1 showed a strong positive relation between community education and adequate ANC visits. The likelihood of having complete ANC visits was higher for women resided in highly literate communities than the women resided in communities with medium and lower portion of literate women. This pattern of association was consistent with the findings from two African countries Nigeria and $\mathrm{Mali}^{23,27}$. After controlling for other community characteristics in Model 3 and individual level characteristics in Model 4, community education continued to exhibit significant positive relationship with ANC visits which implied the relative importance of community education on adequate ANC visits. It suggested that with high educational attainment, women have more autonomy and material resources to access health care services ${ }^{22}$.

Women from urban areas were found to be well ahead in ANC visits than women from rural areas and similar results were also observed in some of previous studies ${ }^{15}$. More accessible health care facilities in urban areas compared to that in rural areas can justify such effect. Women from Rangpur were ahead on adequate ANC visits compared to Khulna and women from all other regions are lagged behind on ANC visits. These regional differentials were in alignment with The United Nations International Children's Emergency Fund(UNICEF) report $^{27}$. Individual level characteristics such as mother's age at last birth, birth order, household wealth, husband's education, media access and decision on health care were found to have statistically significant adjusted effects on ANC visits. Younger women (aged < 20) were less likely to have adequate ANC visits and previous studies confirmed this finding ${ }^{19}$. It was observed that women had higher ANC visits for their first birth than subsequent higher order births. The higher number of children creates pressure on household resources which makes negative impact on number of ANC visits ${ }^{13}$. Mothers from rich households were more likely to have adequate ANC visits and this compared well with findings from previous studies $^{13,19-20}$. It was also observed that husband's 
education had a positive impact on women's ANC visits behavior. Few studies in Africa confirmed this finding ${ }^{26}$. Furthermore, media access and decision making on seeking health care services were also found to be positively related with adequate ANC visits and several studies on maternal health care showed similar results ${ }^{14,20}$.

To reduce maternal mortality and pregnancy related complications, adequate antenatal care visits can play an important role. Findings from this study suggests that individual level factors as well as community level factors, especially community education are strongly associated with higher rate of ANC visits. Thus, increasing women's education in unprivileged and disadvantaged communities can play a prominent role in increasing the number of ANC visits which as a result can reduce maternal mortality and complications.

\section{Acknowledgement}

The authors are thankful to National Institute of Population Research and Training (NIPORT), Bangladesh for providing the BDHS 2014 data. The authors also express their gratitude to Dr. Wasimul Bari, Professor of Statistics at the University of Dhaka, for his valuable suggestions in preparing this manuscript.

\section{References}

1. WHO, 2015. Health in 2015 from MDGs to SDGs. World Health Organization, Retrieved August 15, 2018, http://www.who.int/gho/publications/mdgs-sdgs/en

2. WHO, 2012. MDG 5: Maternal and reproductive health: Women by country. World Health Organization. Retrieved November 14, 2013, http://apps.who.int/gho/data/node.main.531

3. National Institute of Population Research and Training (NIPORT), International Centre for Diarrhoeal Disease Research, Bangladesh (icddr,b), and MEASURE Evaluation. 2003. Bangladesh Maternal Mortality and Health Care Survey 2001: Preliminary Report. Dhaka, Bangladesh, and Chapel Hill, NC, USA: NIPORT, icddr,b, and MEASURE Evaluation.

4. National Institute of Population Research and Training (NIPORT), International Centre for Diarrhoeal Disease Research, Bangladesh (icddr,b), and MEASURE Evaluation. 2012. Bangladesh Maternal Mortality and Health Care Survey 2010: Preliminary Report. Dhaka, Bangladesh, and Chapel Hill, NC, USA: NIPORT, icddr,b, and MEASURE Evaluation.

5. National Institute of Population Research and Training (NIPORT), International Centre for Diarrhoeal Disease Research, Bangladesh (icddr,b), and MEASURE Evaluation. 2017. Bangladesh Maternal Mortality and Health Care Survey 2016: Preliminary Report. Dhaka, Bangladesh, and Chapel Hill, NC, USA: NIPORT, icddr,b, and MEASURE Evaluation.

6. WHO, 2018. SDG 3: Ensure healthy lives and promote wellbeing for all at all ages. Sustainable Development Goals, Retrieved 10 September, 2018, http://www.who.int/sdg/ targets/en/

7. Unicef, 2018. Antenatal Care. Retrieved 15 September, 2018, https://data.unicef.org/topic/maternal-health/antenatal-care/

8. Titaley, C. R., C. L. Hunter, P. Heywood, and M. J. Dibley, 2010. Why don't some women attend antenatal and postnatal care services?: a qualitative study of community members' perspectives in Garut, Sukabumi and Ciamis districts of West
Java Province, Indonesia. BMC Pregnancy and Childbirth, 10, 61. doi:10-1186/1471-2393-10-61

9. Pandit R. D., 1992. Role of Antenatal Care in Reducing Maternal Mortality, Asia-Oceania J. Obstet. Cynaecol, 18(1), 1-6.

10. Health Population and Nutrition Sector Development Program (HPNSDP), 2011-16. Retrieved from http://www. mohfw.gov.bd/index.php?option=com_content $\&$ view=article \&id=166\&Itemid=150\&lang=en

11. Bangladesh Demographic and Health Survey (BDHS), 2014. NIPORT, Dhaka, Bangladesh; Mitra and Associates, Dhaka, Bangladesh.

12. Nisar, N. and F. White, 2003. Factors affecting utilization of antenatal care among reproductive age group women (15-49 years) in an urban squatter settlement of Karachi. Journal of the Pakistan Medical Association, 53(2), 47-53.

13. Jayaraman, A., S. Chandrasekhar, and T. Gebreselassie, 2008. Factors Affecting Maternal Health Care Seeking Behavior in Rwanda. Demographic and Health Research, 59, 1-31.

14. Navaneetham, K. and A. Dharmalingam, 2002. Utilization Of Maternal Health Care Services In South India. Social Science \& Medicine, 55, 1849-1869.

15. Mohammad, K. A., F. T. Zahura, and M. M. Rahman, 2017. Importance of Maternal Education on Antenatal Care Visits in Bangladesh. Bangladesh J. Sci. Res, 30(1\&2), 23-33.

16. Becker S., D. H. Peters, R. H., C. Gultiano, and R.E. Black, 1993. The determinants of use of maternal and child health services in Metro Cebu, the Philippines. Health Transition Review, 3, 77-89

17. Matsumura, M. and B. Gubhaju, 2001. Women's status household structure and the utilisation of maternal health services in Nepal. Asia-Pacific Population Journal, 16(1), 23-44.

18. Raghupathy, S., 1996. Education and the use of maternal health care in Thailand. Social and Medicine, 43(4) 459-471.

19. Rahman, K. M. M., 2009. Determinants of Maternal Health Care Utilization in Bangladesh. Research Journal of Applied Sciences, 4, 113-119.

20. Chakraborty, N., M. A. Islam, R. I. Chowdhury, W. Bari, and H. H. Akhter, 2003. Determinants of the use of maternal health services in rural Bangladesh. Health Promotion International, 18(4), 327-337

21. Jat, T. R., N. Ng and M. S. Sebastian, 2011. Factors affecting the use of maternal health services in Madhya Pradesh state of India: a multilevel analysis. International Journal for Equity in Health, 10(59). doi : 10-1186/1475-9276-10-59

22. Ononokpono, D. N. and C. O. Odimegwu, 2014. Determinants of Maternal Health Care Utilization in Nigeria: A Multilevel Approach. Pan African Medical Journal, 17(1), 2. doi : 1011694/pamj.supp.2014.17.1.3596

23. Dahiru, T., and O. M. Oche, 2015. Determinants of antenatal care, institutional delivery and postnatal care services utilization in Nigeria. Pan African Medical Journal, 21, 321. doi : 10-11604/pamj.2015.21.321-6527

24. Afulani, P. A. 2015. Rural/Urban and Socioeconomic Differentials in Quality of Antenatal Care in Ghana. PLoS ONE, 10(2), e0117996. doi:10.1371/journal.pone.

25. Yebyo, H., M. Alemayehu and A. Kahsay, 2015. Why Do Women Deliver at Home? Multilevel Modeling of Ethiopian National Demographic and Health Survey Data. PLoS ONE, 10(4), e0124718. doi:10.1371/journal.pone.0124718

26. Gage, A. J., 2007. Barriers to the utilization of maternal health care in rural Mali. Soc Sci Med, 65(8), 1666-1682.

27. Unicef, 2016. Maternal and Newborn Health Disparities in Bangladesh. Unicef for every child. https://data.unicef.org/ content/uploads/country_profiles/Bangladesh/country\%20profil e_BGD 